POS PROCEEDINGS

\title{
Pion quasi parton distribution function on a fine lattice
}

Charles Shugert, Taku Izubichi, Luchang Jin, Christos Kallidonis, Nikhil Karthik, Swagato Mukherjee, Peter Petreczky, Sergey Syritsyn

E-mail: charles.shugertestonybrook.edu

\begin{abstract}
We present a calculation of the bare quasi-PDF (qPDF) of the pion. We perform these calculations using the HotQCD HISQ gauge ensemble for our sea quarks along with a Wilson-Clover valence quark action. Our lattice size is $48^{3} \times 64$, our lattice spacing is set at a $=0.06 \mathrm{fm}$, and our pion mass is tuned to $300 \mathrm{MeV}$. Utilizing momentum smearing techniques, we compute the bare qPDF boosted up to momentum $1.72 \mathrm{GeV}$. In addition we explore excited state contamination of the three-point correlator.
\end{abstract}

The 36th Annual International Symposium on Lattice Field Theory - LATTICE2018

22-28 July, 2018

Michigan State University, East Lansing, Michigan, USA. 


\section{Introduction}

Deep inelastic scattering experiments can be used to probe the internal structure of hadrons. The total cross-section in these interactions can be written as a convolution of a partonic crosssection which can be computed perturbativly, and a parton distribution function (PDF) defined as

$$
q(\xi)=\frac{1}{4 \pi} \int d \xi^{-} e^{i x P^{+} \xi^{-}}\left\langle N(P)\left|\bar{\psi}\left(\xi^{-}\right) \gamma^{+} W_{L}\left(\xi^{-}, 0\right) \psi(0)\right| N(P)\right\rangle,
$$

where $\mid N(P\rangle$ is the hadron state, $W_{L}\left(\xi^{-}, 0\right)=e^{i g \int_{0}^{\xi^{-}} d \xi^{-} A^{+}}$is a straight Wilson Line on the lightcone, and $\xi^{ \pm}=(t \pm z) / \sqrt{2}$. PDFs, defined along the light-cone, are inaccessible from direct lattice simulations due to the sign problem. Quasi-PDFs have been proposed as an alternative method towards extracting PDFs, in which the quark and anti-quark in the operator are only spatially separated [1].

$$
q\left(x, P_{z}\right)=\frac{1}{4 \pi} \int d z e^{-i x P^{z} z}\left\langle N(P)\left|\bar{\psi}(z) \Gamma W_{L}(z, 0) \psi(0)\right| N(P)\right\rangle,
$$

where $\Gamma$ is a gamma matrix is either $\gamma_{z}$ or $\gamma_{t}$. So long as the hadron is highly boosted, matching the qPDF to the PDF can be done using large momentum effective field theory [2].

Here we present calculations of the bare qPDF matrix element for a $300 \mathrm{MeV}$ pion. Our lattice size is $48^{3} \times 64$ and our lattice spacing is $0.06 \mathrm{fm}$. We use a mixed action using a HotQCD HISQ gauge ensemble [3] and a Wilson-Clover quark action with one level of HYP smearing [4]. In section two, we study excited state effects on the two-point correlator under different smearing schemes. We also examine the dispersion relation of the pion extracted from a two-state fit of the two-point correlator to study lattice artifacts at large momentum. In section three, we explore two methods, the summation method and a two-state fit, that remove excited states from the threepoint correlator. For all matrix elements we used All-Mode Averaging (AMA) [5] with 32 sloppy calculations to one exact solve for each configuration.

\section{Two-Point Function Analysis}

Here we present analysis of excited states and systematic error arising from the two-point correlators. We first look at the effective mass plots at zero momentum under different amounts of Wuppertal Smearing steps [6]. To save computation time, we switch to Coulomb gauge-fixed Gaussian smearing with a width set to match the optimal Wuppertal smearing. We compute effective masses by solving the following nonlinear equation

$$
C_{2 p t}\left(t+1 ; P_{z}\right) / C_{2 p t}\left(t ; P_{z}\right)=\frac{\cosh \left(M_{\mathrm{eff}}\left(T / 2-t+1 ; P_{z}\right)\right)}{\cosh \left(M_{\mathrm{eff}}\left(T / 2-t ; P_{z}\right)\right)},
$$

where $T$ is the length of the time-direction on our lattice, $t$ is a given time-slice on the lattice, and $E$ is the effective mass. Figure 1. shows our results. We can see that the effective mass plot reaches a plateau faster with 90 Wuppertal smearing than with only 40 steps. Furthermore, we find that appropriate Gaussian smearing in Coulomb gauge has the same signal as 90 steps of Wuppertal Smearing. For the width of the Gaussian we use is $0.31 \mathrm{fm}$. 
Table 1: Energy Gap vs $P_{z}$

\begin{tabular}{|c|c|c|c|c|}
\hline$P_{z}\left(\mathrm{~N}_{\mathrm{cfg}}\right)$ & $0 \mathrm{GeV}(52)$ & $0.86 \mathrm{GeV}(168)$ & $1.29 \mathrm{GeV}(168)$ & $1.72 \mathrm{GeV}(168)$ \\
\hline$\Delta E_{2,1}$ & $1.39(38) \mathrm{GeV}$ & $1.26(04) \mathrm{GeV}$ & $1.15(08) \mathrm{GeV}$ & $1.32(36) \mathrm{GeV}$ \\
\hline
\end{tabular}

As the signal of the two-point function deteriorates with larger momentum, we turn to boosted sources, as prescribed in Ref. [7] for Coulomb gauge-fixed Gaussian smearing. Here the valence quarks are boosted to momentum $\vec{k}=\zeta \vec{P}$, where $\vec{P}$ is the momentum of our pion and $\zeta$ is the momentum fraction of the quark. In Figure 2. we show effective mass plots of our pion using boosted-smeared sources for different values of $\zeta$ at momentum $0.86 \mathrm{GeV}, 1.29 \mathrm{GeV}$, and 1.72 $\mathrm{GeV}$. At momentum $0.86 \mathrm{GeV} \zeta=1.0$ yields data with smaller error bars and smaller fluctuations about the expected mass from the dispersion relation. At $1.29 \mathrm{GeV}, \zeta=1.0$ is too large, and $\zeta=0.67$ yields the best results. At $1.72 \mathrm{GeV}, \zeta=0.5$ begins to fluctuate wildly early on, whereas $\zeta=0.75$ and $\zeta=1.00$ are comparable to each other. Despite this the error bars grow quickly and more statistics are needed at the momentum. For momentum larger than $1 \mathrm{GeV}$ however, we must omit data with $\zeta=0$ as it becomes too noisy to plot. And so, for large momentum calculations, it is necessary to have large enough $\zeta$ in order to have a clean signal.

Next we study how well the pion with appropriate boosted smearing follows the expected dispersion relation $E^{2}\left(P_{z}^{2}\right)=P_{z}^{2}+m_{\pi}^{2}$. Here we fit our two-point correlator data at fixed momentum to the following form:

$$
C_{2 \mathrm{pt}}\left(P_{z}, t\right)=\sum_{i=1}^{2} 2 A_{i} e^{-\frac{1}{2} E_{i} T} \cosh \left(E_{i}(T / 2-t)\right)
$$

where $T$ is the time extent of the lattice, $t$ is the source-sink separation, $A_{i}=|\langle i \mid \pi\rangle|^{2}$, and $E_{i}$ is the $i$ 'th energy eigenstate. Figure 2 shows our results, and we find that there is no deviation from our extracted masses and the expected dispersion relation of the pion. Table 1. shows $\Delta E_{2,1}=E_{2}-E_{1}$ for momentum $0 \mathrm{GeV}, 0.86 \mathrm{GeV}, 1.29 \mathrm{GeV}$, and $1.72 \mathrm{GeV}$.

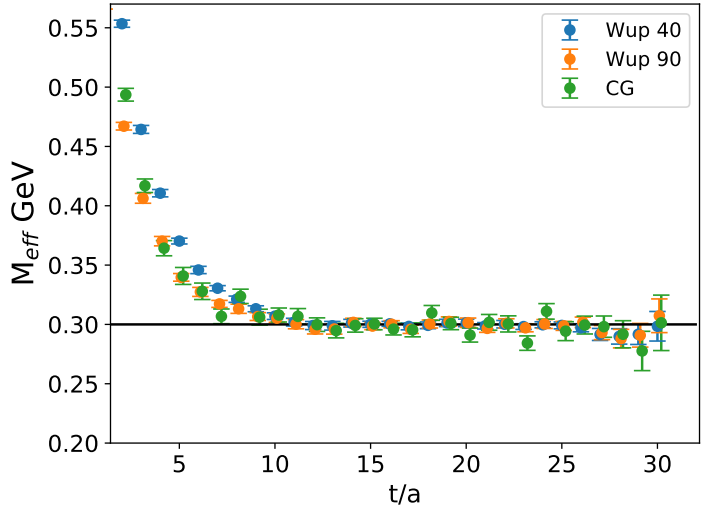

Figure 1: Effective Mass Plots at $P_{z}=0 \mathrm{GeV}$ using 50 configurations. Wup 40(90) correspond to 40(90) Wuppertal Smearing steps. CG stands for Coulomb gauge-fixed Gaussian smearing.

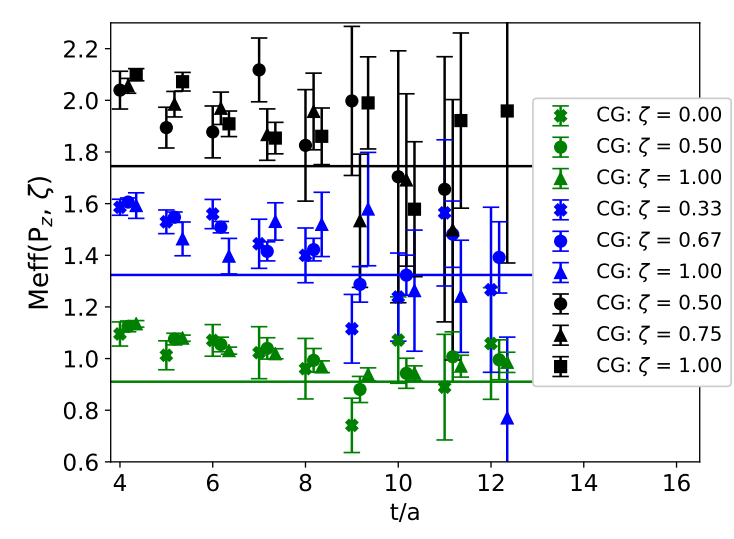

Figure 2: $\mathrm{M}_{\mathrm{eff}}\left(\mathrm{P}_{z}\right)$ vs different values of $\zeta$ with 50 configurations, Green, blue, and black points correspond to momentum $0.86,1.29$, and 1.72 $\mathrm{GeV}$ respectively. 


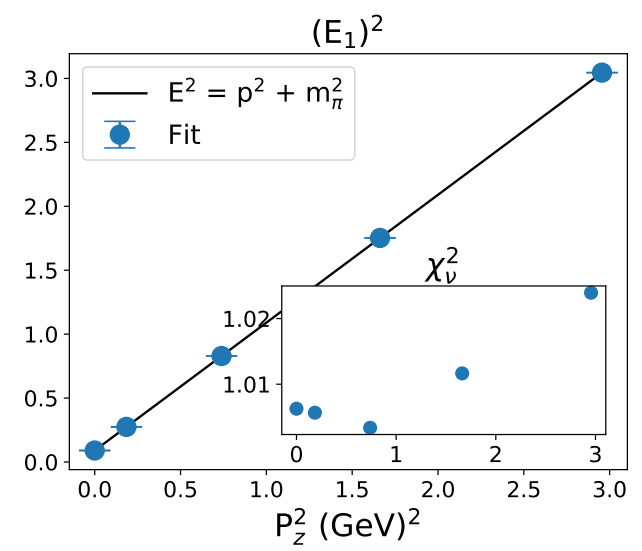

Figure 3: Ground-state energy squared vs $P_{z}^{2}$. Ground-state exctracted from fit to Eq (2.2).

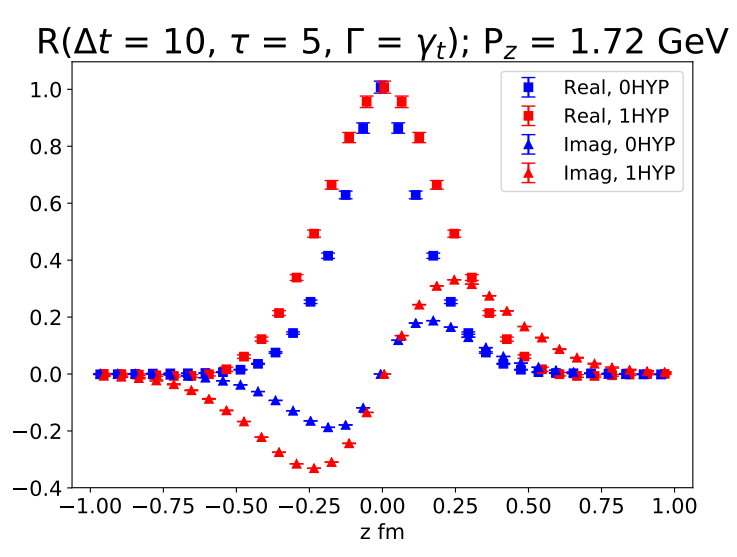

Figure 4: Ratio defined by Eq. (3.1) vs z under different applications of HYP smearing.

\section{Three-Point Function Analysis}

To extract the ground state calculation of the three point function, we form the ratio to remove the overlap factor between the source-operator and the pion state

$$
R(\Delta t, \tau z ; \Gamma)=\frac{\left\langle\pi(p, \Delta t) \mathscr{O}_{\Gamma}(z, \tau) \bar{\pi}(0)\right\rangle}{\langle\pi(p, \Delta t) \pi \bar{\pi}(0)\rangle}=\frac{\sum_{n, n^{\prime}} A_{n} A_{n^{\prime}}^{*} e^{-E_{n} \Delta t} e^{-\left(E_{n^{\prime}}-E_{n}\right) \tau}\left\langle n\left|\mathscr{O}_{\Gamma}(z)\right| n^{\prime}\right\rangle}{\sum_{m}\left|A_{m}\right|^{2} e^{-E_{m} \Delta t}}
$$

where $A_{n}=\langle\pi \mid n\rangle, \mathscr{O}_{\Gamma}(z, \tau)=\bar{\psi}(z, \tau) \Gamma W_{L}(z, 0) \psi(0), \Delta t$ is the source-sink separation, and $\tau$ is the operator insertion time-slice, such that $0<\tau<\Delta t$. Expanding this to the second energy eigenstate yields

$$
R(\Delta t, \tau, z ; \Gamma) \sim \frac{\mathcal{M}(z)+\mathcal{A}(z) e^{-\Delta E_{2,1} \tau}+\mathcal{A}^{\dagger}(z) e^{-\Delta E_{2,1}(\Delta t-\tau)}+\mathcal{B}(z) e^{-\Delta E_{2,1} \Delta t}+\ldots}{1+\mathcal{C} e^{-\Delta E_{2,1} \Delta t}+\ldots}
$$

Here, $\mathcal{M}(z)=\left\langle 1\left|\mathscr{O}_{\Gamma}(z)\right| 1\right\rangle$ is the desired quantity, $\mathcal{A}(z)=\frac{A_{1} A_{2}^{*}}{\left|A_{1}\right|^{2}}\left\langle 1\left|\mathscr{O}_{\Gamma}(z)\right| 2\right\rangle, \mathcal{B}(z)=\mathcal{C}\left\langle 2\left|\mathscr{O}_{\Gamma}(z)\right| 2\right\rangle$, and $\mathcal{C}=\frac{\left|A_{2}\right|^{2}}{\left|A_{1}\right|^{2}}$, a term suppressed for large $\Delta E_{2,1} \Delta t$.

In Figure 4 we display a comparison of our bare quasi-PDF matrix element when we apply 0 and 1 HYP smearing operations onto the Wilson Line of our operator [4]. We do so to see whether or not lattice artifacts due to short distance fluctuations of the gauge field can be smeared out with HYP smearing. Here $\Delta t=10$, and the operator insertion $\tau=\Delta t / 2$. What we see is that the matrix element becomes wider and larger with respect to the length of the Wilson Line. This is expected, as the self energy divergence from the Wilson Line decreases with HYP smearing. However, the statistical error in the matrix element does not improve much with HYP smearing.

The data in Figure 5 showcases data with 1 HYP-Smeared Wilson Line. Points on the same $\mathrm{z}$-value are shifted horizontally for better visability. In addition all $\mathrm{z}$ values greater than $0.48 \mathrm{fm}$ in magnitude are computed with 52 configurations, whereas all smaller $\mathrm{z}$ values are computed using 168 configurations. Here we plot the ratio vs the length of the Wilson Line with respect to $\Delta t=8$, 10 , and 12 , with $\tau=\Delta t / 2$. On one hand the error in smaller $\Delta t$ are very small and increases with increasing $\Delta t$. On the other hand, excited state contamination dies off exponentially with larger 
source-sink separation. And so, to extract the ground-state quasi-PDF matrix element, we employ two fitting procedures used in references $[8,9]$.
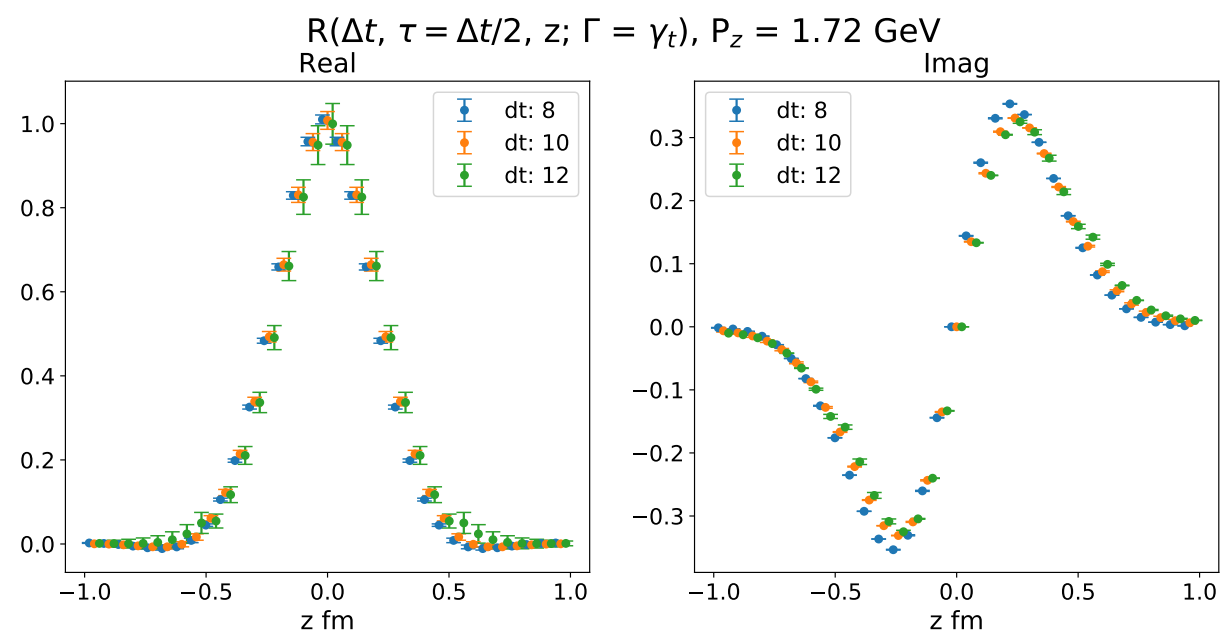

Figure 5: Ratio defined by Eq. (3.1) vs z at $\Delta t 8$ (blue), 10 (orange), and 12 (green). $\tau=\Delta t / 2$.

The first method employed is the summation method [9]. Here one takes the ratio and sums over all operator insertions minus a certain number of end points. Here we sum the ratio excluding $\tau>\left|\tau_{o}\right|$. The summed ratio takes the following form

$$
R_{\text {sum }}(\Delta t, z ; \Gamma)=\sum_{\tau=\tau_{o}}^{\Delta t-\tau_{o}} R(\Delta t, \tau, z ; \Gamma) \sim\left(\mathcal{M}+\mathcal{B} e^{-\Delta E_{2,1} \Delta t}\right)\left(\Delta t-2 \tau_{o}\right)+C,
$$

where $\tau_{o}$ represents the time slices truncated from the sum. For each source-sink separation, we compute the summed ratio and plot it with respect to $\Delta t-2 \tau_{o}$. From there we perform a linear fit to extract the slope, which gives an estimate of $\mathcal{M}$ for large enough $\Delta E_{2,1} \Delta t$. In figures 6 and 7 , we plot the results of the summation method for the ratio evaluated at $\mathrm{z}=0 \mathrm{fm}$ and $\mathrm{z}=0.24 \mathrm{fm}$ using $\tau_{o}=1$ and $\tau_{o}=2$ to determine which gave the more precise result. In both cases $\tau_{o}=2$ gives the most precise result. As this method does not remove some of the systematic error from excited states, we also try a second method. The second method relies on a simultaneous fit of both the two-point and three-point correlator, which we refer to as the two-state fit [8]. Using $\Delta E_{2,1}$ obtained from fitting the two-point correlator, we use all operator insertions excluding end points as well as all available source-sink separation data to fit $\mathcal{M}, \mathcal{A}$, and $\mathcal{B}$ in equation (3.2).

On Figure 8 we plotted the results of the two-state fit and the summation method along with the ratio at $\Delta t=10$ with the $\tau=\Delta t / 2$. Again, the data contains a Wilson Line under one application of HYP smearing. All $\mathrm{z}$ values greater than $0.48 \mathrm{fm}$ in magnitude are computed with 52 configurations and the rest with 168 configurations, with points on the same z-value shifted horizontally for better visability. In addition all the points on the same z-value are shifted horizontally for better visability. We see that almost consistently the results from the summation method give larger error. Data beyond $0.48 \mathrm{fm}$ yields larger error, consistent with the smaller configurations used compared to data with $\mathrm{z}<0.48 \mathrm{fm}$. The imaginary part of the summation method results does differ somewhat to the imaginary part of the two-state fit, meaning that excited states have some effects in the imaginary part. 


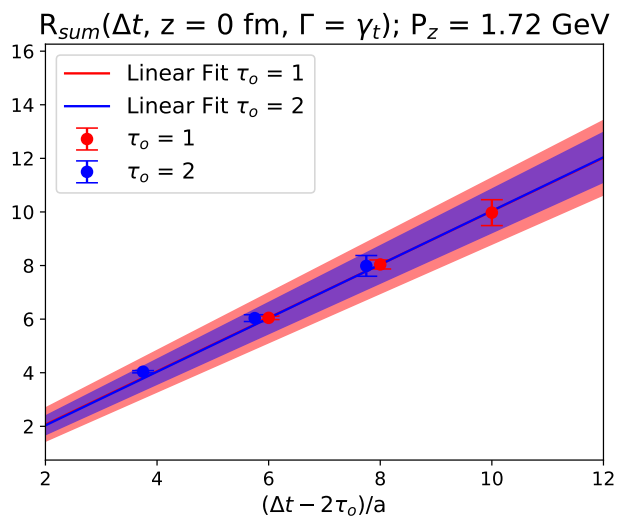

Figure 6: Summation method results for $\mathrm{z}=0 \mathrm{fm}$. Red and blue data are for results with $\tau_{o}=1$ and $\tau_{o}=2$ respectively. The line and bands about the line correspond to the results of the linear fit and the error bands to that fit result.

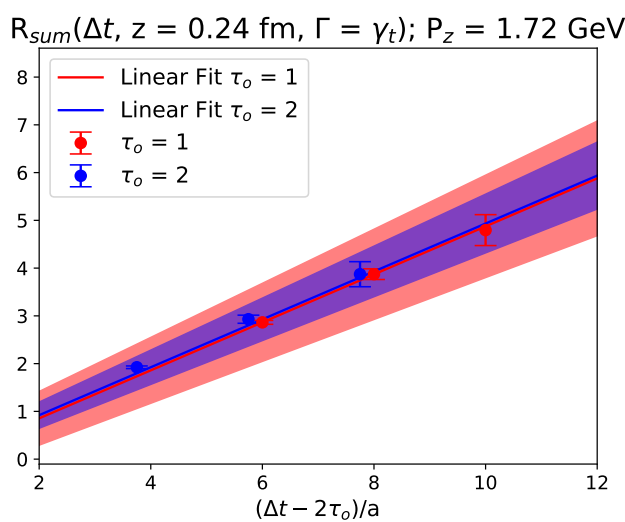

Figure 7: Summation method results for $\mathrm{z}$ $=0.24 \mathrm{fm}$. Red and blue data are for results with $\tau_{o}=1$ and $\tau_{o}=2$ respectively. The line and bands about the line correspond to the results of the linear fit and the error bands to that fit result.
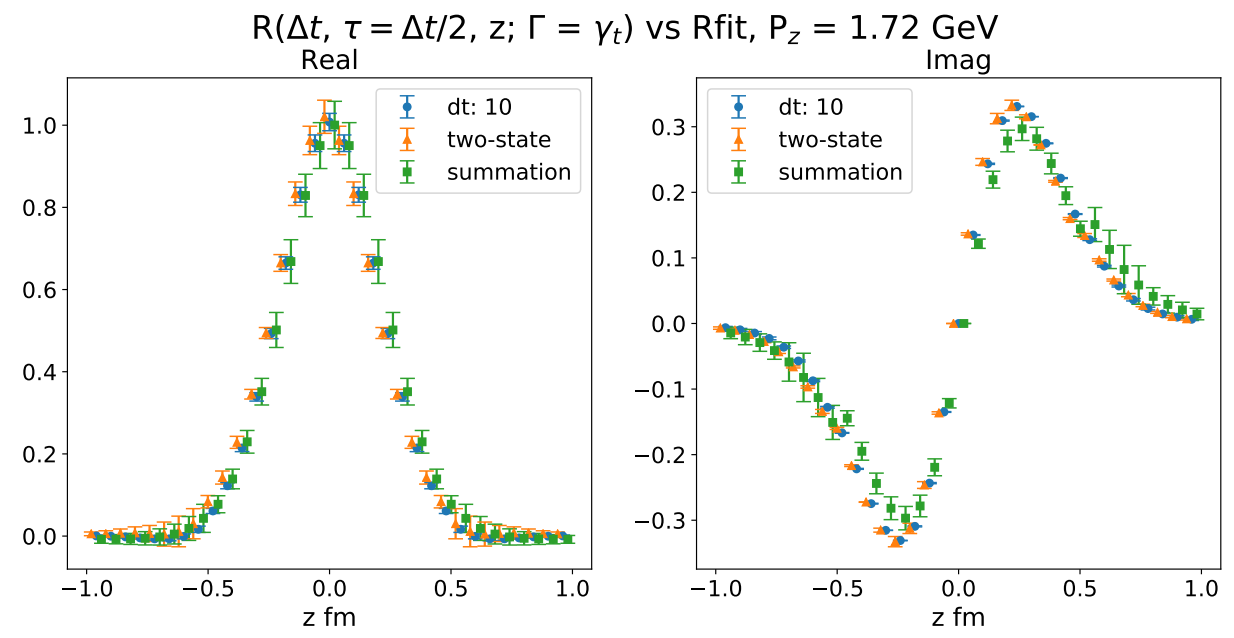

Figure 8: Ratio defined by Eq. (3.1) vs z at $\Delta t=10 \tau=\Delta t / 2$ (blue), extracted ground state matrix element from a two-state fit (orange), and extracted ground state matrix element from the summation method.

\section{Conclusion}

We have presented the calculation of the bare quasi-PDF matrix element at momentum 1.72 $\mathrm{GeV}$ for a lattice spacing $0.06 \mathrm{fm}$ using a mixed HISQ-sea and a $1 \mathrm{HYP}$-smeared valence WilsonClover fermion action for a $300 \mathrm{MeV}$ pion. In order to maximally project our pion field onto the ground state, we studied different smearing techniques. We found optimal Gaussian sources to project onto the pion ground state, and have increased the signal of our data at large momentum using boosted sources. We also studied the ground state energy of the pion, extracted from a double exponential fit up to momentum $1.72 \mathrm{GeV}$, and found that agrees with the expected dispersion relation $E^{2}=p^{2}+m_{\pi}^{2}$. Regarding the quasi-PDF matrix element, we looked at the HYP-smearing 
dependence. Furthermore we explored excited state removal of our three-point correlator using a simultaneous fit of the two and three-point correlator and by applying the summation method.

\section{Acknowledgments}

C.S, T.I, S.M, P.P. and N.K. acknowledge support by the U.S. Department of Energy under contract No. DE-SC0012704, BNL LDRD project No. 16-37 and Scientific Discovery through Advance Computing (SCIDAC) award "Computing the Properties of Matter with Leadership Computing Resources". The computations were carried out using USQCD facilities at JLab and BNL under a USQCD type-A project. This research also used an award of computer time provided by the INCITE program at the Oak Ridge Leadership Computing Facility, which is a DOE Office of Science User Facility supported under Contract DE-AC05-00OR22725.

\section{References}

[1] X. Ji, Parton Physics on a Euclidean Lattice, Phys. Rev. Lett. 110 (2013) 262002 [1305 . 1539].

[2] X. Ji, Parton Physics from Large-Momentum Effective Field Theory, Sci. China Phys. Mech. Astron. 57 (2014) 1407 [1404.6680].

[3] HотQCD collaboration, A. Bazavov et al., Equation of state in ( 2+1)-flavor QCD, Phys. Rev. D90 (2014) 094503 [1407.6387].

[4] A. Hasenfratz, R. Hoffmann and F. Knechtli, The Static potential with hypercubic blocking, Nucl. Phys. Proc. Suppl. 106 (2002) 418 [hep-lat/ 0110168$].$

[5] E. Shintani, R. Arthur, T. Blum, T. Izubuchi, C. Jung and C. Lehner, Covariant approximation averaging, Phys. Rev. D91 (2015) 114511 [1402.0244].

[6] S. Gusken, U. Low, K. H. Mutter, R. Sommer, A. Patel and K. Schilling, Nonsinglet Axial Vector Couplings of the Baryon Octet in Lattice QCD, Phys. Lett. B227 (1989) 266.

[7] G. S. Bali, B. Lang, B. U. Musch and A. Schäfer, Novel quark smearing for hadrons with high momenta in lattice QCD, Phys. Rev. D93 (2016) 094515 [1602 . 05525].

[8] A. Abdel-Rehim et al., Nucleon and pion structure with lattice QCD simulations at physical value of the pion mass, Phys. Rev. D92 (2015) 114513 [1507.04936].

[9] L. Maiani, G. Martinelli, M. L. Paciello and B. Taglienti, Scalar Densities and Baryon Mass Differences in Lattice QCD With Wilson Fermions, Nucl. Phys. B293 (1987) 420. 